\title{
Exceptional Set of Waring-Goldbach Problem with Unequal Powers of Primes
}

\author{
Xiaodong Zhao
}

\begin{abstract}
In this paper, it is proved that with at most $O\left(N^{17 / 42+\varepsilon}\right)$ exceptions, all even positive integer $n, n \in[N / 2, N]$, can be represented in the form $p_{1}^{2}+p_{2}^{2}+p_{3}^{3}+$ $p_{4}^{3}+p_{5}^{4}+p_{6}^{4}$, where $p_{1}, p_{2}, p_{3}, p_{4}, p_{5}, p_{6}$ are prime numbers. This improves a recent result $O\left(N^{13 / 16+\varepsilon}\right)$ due to Zhang and $\mathrm{Li}[13$.
\end{abstract}

\section{Introduction}

Let $n, k_{1}, k_{2}, \ldots, k_{s}$ be natural numbers such that $2 \leq k_{1} \leq k_{2} \leq \cdots \leq k_{s}, n>s$. Waring's problem of mixed type concerns the representation of a natural number $n$ as the form

$$
n=x_{1}^{k_{1}}+x_{2}^{k_{2}}+\cdots+x_{s}^{k_{s}} .
$$

Not very much is known the results of this kind. For references in this aspect, we refer the reader to Section P12 of LeVeques Reviews in number theory, the bibliography in Vaughan [10] and the recent papers by J. Brüdern [2,3] and by T. D. Wooley [12].

In 1970, Vaughan [9] obtained the asymptotic formula for the number of representations of an integer as the sum of two squares, two cube, and two fourth powers. Let $\widetilde{R}(n)$ denote the number of representations of the integer $n$ in the shape

$$
n=x_{1}^{2}+x_{2}^{2}+x_{3}^{3}+x_{4}^{3}+x_{5}^{4}+x_{6}^{4}
$$

with $x_{i} \in \mathbb{N}(1 \leq i \leq 6)$, and let

$$
\Theta_{2,3,4}(n)=\sum_{q=1}^{\infty} \frac{1}{q^{6}} \sum_{\substack{a=1 \\(a, q)=1}}^{q} \prod_{i=1}^{3}\left(\sum_{x_{i}=1}^{q} e\left(\frac{a x_{i}^{i+1}}{q}\right)\right)^{2} e\left(-\frac{a n}{q}\right) .
$$

Hence, Vaughan [9] proved that

$$
\widetilde{R}(n)=\frac{\Gamma^{2}\left(\frac{3}{2}\right) \Gamma^{2}\left(\frac{4}{3}\right) \Gamma^{2}\left(\frac{5}{4}\right)}{\Gamma\left(\frac{13}{6}\right)} \Theta_{2,3,4}(n) n^{7 / 6}+O\left(n^{7 / 6-1 / 96+\varepsilon}\right) .
$$

Received May 27, 2019; Accepted October 13, 2019.

Communicated by Yu-Ru Liu.

2010 Mathematics Subject Classification. Primary: 11P32; Secondary: 11P05, 11P55.

Key words and phrases. Waring-Goldbach problem, circle method, exceptional set. 
In view of Vaughan's result, it is reasonable to propose the conjecture that every sufficiently large even integer $n$ can be expressed as the sum of two squares, two cube and two fourth powers of primes. That is, for sufficiently large even integer $n$, the equation

$$
n=p_{1}^{2}+p_{2}^{2}+p_{3}^{3}+p_{4}^{3}+p_{5}^{4}+p_{6}^{4}
$$

is solvable in primes $p_{j}(1 \leq j \leq 6)$. Here and in the sequel, the letter $p$, with or without subscripts, always stands for a prime number. This conjecture is perhaps out of reach at present times. It is possible, however, to obtain a weaker result with $p_{1}$ replaced by an almost-prime. Let $\mathcal{P}_{r}$ denote an almost-prime with at most $r$ prime factors, counted according to multiplicity. In 2015, Lü 7] proved that for every sufficiently large even integer $n$, the equation

$$
n=x^{2}+p_{2}^{2}+p_{3}^{3}+p_{4}^{3}+p_{5}^{4}+p_{6}^{4}
$$

is solvable with $x$ being an almost-prime $\mathcal{P}_{6}$. On the other hand, in 2017, Liu [6] proved that every sufficiently large even integer $n$ can be represented as two squares of primes, two cubes of primes, two fourth powers of primes and 41 powers of 2 , i.e.,

$$
n=p_{1}^{2}+p_{2}^{2}+p_{3}^{3}+p_{4}^{3}+p_{5}^{4}+p_{6}^{4}+2^{v_{1}}+\cdots+2^{v_{41}} .
$$

Let $E(N)$ denote the number of positive even integer $n, n \in[N / 2, N]$, which can not be represented as (1.1). In 2018, Zhang and Li [13] considered the exceptional set of the problem 1.1) and got

$$
E(N) \ll N^{13 / 16+\varepsilon}
$$

In this paper, we sharpen the above result and establish the following result.

Theorem 1.1. Let $E(N)$ be defined as above. Then, for any $\varepsilon>0$, we have

$$
E(N) \ll N^{17 / 42+\varepsilon}
$$

Remark 1.2. Note that $13 / 16 \approx 0.8125$ and $17 / 42 \approx 0.4048$, Theorem 1.1 improves the result of Zhang and Li [13] to approximately half of the original. We establish Theorem 1.1 by means of the circle method in combination with some new ideas of Liu [5] and Zhang and Li [13]. Especially, the method from Liu [5] plays an important role in dealing with the minor arcs.

Actually, the method of Liu [5] derives from Wooley [11]. Utilizing this method and combining with the key Lemma 2.2 , we obtain the better estimates about $\mathfrak{m}_{1}$ and $\mathfrak{m}_{2}$ than the results of [13]. Consequently, we can make this improvement.

Notations. Throughout this paper, $\varepsilon$ and $A$ always denote positive constants which are arbitrary small and sufficiently large, respectively, which may not be the same at different 
occurrences. $e(x)=e^{2 \pi i x} ; f(x) \ll g(x)$ means that $f(x)=O(g(x)) ; f(x) \asymp g(x)$ means that $f(x) \ll g(x) \ll f(x)$. $N$ is a sufficiently large integer, and thus we use $L$ to denote both $\log N$ and $\log n$. The letter $c$, with or without subscripts or superscripts, always denotes a positive constant.

\section{Outline of method}

Throughout, we assume that $N$ is a sufficiently large positive integer. In order to apply the circle method, we set

$$
P=N^{9 / 80-2 \varepsilon}, \quad Q=N^{71 / 80+\varepsilon} .
$$

For any integers $a, q$ satisfying

$$
1 \leq a \leq q \leq Q, \quad(a, q)=1
$$

by Dirichlet's lemma on rational approximation (see Lemma 2 on page 142 of Karatsuba [4]), we define the major arc $\mathfrak{M}$ and minor arc $\mathfrak{m}$ as usual, namely

$$
\mathfrak{M}=\bigcup_{1 \leq q \leq P} \bigcup_{\substack{1 \leq a \leq q \\(a, q)=1}} \mathfrak{M}(a, q), \quad \mathfrak{m}=\left[\frac{1}{Q}, 1+\frac{1}{Q}\right] \backslash \mathfrak{M},
$$

where

$$
\mathfrak{M}(a, q)=\left\{\alpha \in[0,1]:\left|\alpha-\frac{a}{q}\right| \leq \frac{1}{q Q}\right\} .
$$

For $k=2,3,4$, we set

$$
f_{k}(\alpha)=\sum_{X_{k}<p \leq 2 X_{k}}(\log p) e\left(p^{k} \alpha\right)
$$

where $X_{k}=\left(\frac{N}{16}\right)^{1 / k}$. Let

$$
R(n)=\sum_{\substack{n=p_{1}^{2}+p_{2}^{2}+p_{3}^{3}+p_{4}^{3}+p_{5}^{4}+p_{6}^{4} \\ X_{2}<p_{1}, p_{2} \leq 2 X_{2}, X_{3}<p_{3}, p_{4} \leq 2 X_{3}, X_{4}<p_{5}, p_{6} \leq 2 X_{4}}}\left(\log p_{1}\right) \cdots\left(\log p_{6}\right)
$$

Then

$$
\begin{aligned}
R(n) & =\int_{0}^{1}\left(\prod_{k=2}^{4} f_{k}^{2}(\alpha)\right) e(-n \alpha) d \alpha=\int_{1 / Q}^{1+1 / Q}\left(\prod_{k=2}^{4} f_{k}^{2}(\alpha)\right) e(-n \alpha) d \alpha \\
& =\left\{\int_{\mathfrak{M}}+\int_{\mathfrak{m}}\right\}\left(\prod_{k=2}^{4} f_{k}^{2}(\alpha)\right) e(-n \alpha) d \alpha .
\end{aligned}
$$


In order to describe the contribution from the major arcs, we introduce some notations. Let

$$
C_{k}(q, a)=\sum_{\substack{m=1 \\(m, q)=1}}^{q} e\left(\frac{a m^{k}}{q}\right) \text { and } B(n, q)=\sum_{\substack{a=1 \\(a, q)=1}}^{q} e\left(-\frac{a n}{q}\right) \prod_{k=2}^{4} C_{k}^{2}(q, a)
$$

The singular series is defined by (see $13,(3.1)]$ )

$$
\Theta(n)=\sum_{q=1}^{\infty} \frac{B(n, q)}{\varphi^{6}(q)}
$$

We define the singular integral as (see $[13,(3.9)]$ )

$$
\Im(n):=\sum_{\substack{m_{1}+\cdots+m_{6}=n \\ X_{2}^{2}<m_{1}, m_{2} \leq\left(2 X_{2}\right)^{2} \\ X_{3}^{3}<m_{3}, m_{4} \leq\left(2 X_{3}\right)^{3} \\ X_{4}^{4}<m_{5}, m_{6} \leq\left(2 X_{4}\right)^{4}}}\left(m_{1} m_{2}\right)^{-1 / 2}\left(m_{3} m_{4}\right)^{-1 / 2}\left(m_{5} m_{6}\right)^{-1 / 2} .
$$

Lemma 2.1. (see [13, Proposition 2.1]) Let the major arcs $\mathfrak{M}$ be defined as in (2.2) with $P$ and $Q$ defined in (2.1). Then, for $n \in[N / 2, N]$ and any $A>0$, there holds

$$
\int_{\mathfrak{M}}\left(\prod_{k=2}^{4} f_{k}^{2}(\alpha)\right) e(-n \alpha) d \alpha=\frac{1}{576} \Theta(n) \Im(n)+O\left(n^{7 / 6} L^{-A}\right),
$$

where $\Theta(n)$ is the singular series defined in (2.4), which is absolutely convergent and satisfies

$$
0<c^{*} \leq \Theta(n) \ll d(n),
$$

where $d(n)$ and $c^{*}$ denote Dirichlet's divisor function and some fixed constant, respectively; while $\Im(n)$ is defined by (2.5) and satisfies

$$
\Im(n) \asymp N^{7 / 6} \text {. }
$$

Next, we need the following lemmas to handle the minor arcs. In this paper, we divide $\mathfrak{m}$ into $\mathfrak{m}_{1}$ and $\mathfrak{m}_{2}$. Let

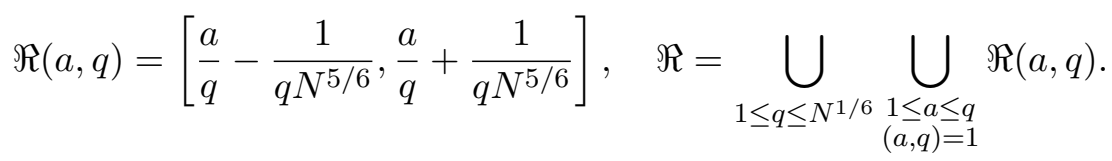

We define

$$
\mathfrak{m}_{1}=\mathfrak{m} \cap \Re, \quad \mathfrak{m}_{2}=\mathfrak{m} \backslash \Re .
$$


Lemma 2.2. Let $f_{2}(\alpha)$ and $f_{4}(\alpha)$ be defined in (2.3). We have

$$
\int_{0}^{1}\left|f_{2}(\alpha) f_{4}^{2}(\alpha)\right|^{2} d \alpha \ll N^{1+\varepsilon}
$$

Proof. This lemma actually derives from Brüdern [1, Lemma 1]. From [1, Lemma 1], we have

$$
\int_{0}^{1}\left|\prod_{i=1}^{s} f_{k_{i}}^{\prime}(\alpha)\right|^{2} d \alpha \ll N^{1 / k_{1}+\cdots+1 / k_{s}+\varepsilon}
$$

where $2 \leq k_{1} \leq \cdots \leq k_{s}$ are natural numbers satisfying

$$
\sum_{i=j+1}^{s} \frac{1}{k_{i}} \leq \frac{1}{k_{j}}, \quad 1 \leq j \leq s-1
$$

and

$$
f_{k}^{\prime}(\alpha)=\sum_{x \leq N^{1 / k}} e\left(\alpha x^{k}\right)
$$

Obviously, when we substitute

$$
f_{k}^{*}(\alpha)=\sum_{p \leq N^{1 / k}} e\left(\alpha p^{k}\right)
$$

for $f_{k}^{\prime}(\alpha), 2.7$ is true.

Utilizing partial summation formula, we know that $f_{k}(\alpha)$ differs from $f_{k}^{*}(\alpha)$ by a $\log N$ (see [8, (29), p. 326]). Hence, taking $k_{0}=1, k_{1}=2, k_{2}=4, k_{3}=4$ satisfying (2.8), we can obtain

$$
\int_{0}^{1}\left|f_{2}(\alpha) f_{4}^{2}(\alpha)\right|^{2} d \alpha \ll N^{1 / 2+1 / 4+1 / 4+\varepsilon}=N^{1+\varepsilon}
$$

Remark 2.3. Lemma 2.2 is very important to estimate the integral over $\mathfrak{m}_{j}, j=1,2$.

Lemma 2.4. (see [13, Lemma 6.6]) Suppose that $\alpha \in \mathfrak{m}_{1}$. Then we have

$$
f_{3}(\alpha) \ll N^{133 / 480+\varepsilon}
$$

Lemma 2.5. (see [13, Lemma 6.7]) Suppose that $\alpha \in \mathfrak{m}_{2}$. Then we have

$$
f_{3}(\alpha) \ll N^{13 / 42+\varepsilon}
$$




\section{Auxiliary estimates}

We are now equipped to establish the auxiliary estimates in this paper, and we initiate our proof by recalling the Farey dissections $(2.2)$ and $(2.6)$ that

$$
R(n)=\left\{\int_{\mathfrak{M}}+\int_{\mathfrak{m}_{1}}+\int_{\mathfrak{m}_{2}}\right\}\left(\prod_{k=2}^{4} f_{k}^{2}(\alpha)\right) e(-n \alpha) d \alpha
$$

where $f_{k}(\alpha)$ is defined in $(2.3)$.

From Lemma 2.1, we can get the evaluation of the integral over $\mathfrak{M}$. Next we will compute the estimation of the integrals over $\mathfrak{m}_{j}, j=1,2$.

\subsection{The integrals over $\mathfrak{m}_{j}, j=1,2$}

We denote by $Z_{j}(N)$ the set of even integers $n, N / 2<n \leq N$, for which the inequality

$$
\left|\int_{m_{j}} f_{2}^{2}(\alpha) f_{3}^{2}(\alpha) f_{4}^{2}(\alpha) e(-\alpha n) d \alpha\right| \geq n^{7 / 6} L^{-A}
$$

holds. For simplicity, we abbreviate the cardinality of $Z_{j}(N)$ to $Z_{j}$. Next, define the complex number $\xi_{j}(n)$ by taking $\xi_{j}(n)=0$ for $n \notin Z_{j}(N)$, and for $n \in Z_{j}(N)$ by means of the equation

$$
\left|\int_{m_{j}} f_{2}^{2}(\alpha) f_{3}^{2}(\alpha) f_{4}^{2}(\alpha) e(-\alpha n) d \alpha\right|=\xi_{j}(n) \int_{m_{j}} f_{2}^{2}(\alpha) f_{3}^{2}(\alpha) f_{4}^{2}(\alpha) e(-\alpha n) d \alpha .
$$

Plainly, one has $\left|\xi_{j}(n)\right|=1$ whenever $\xi_{j}(n)$ is nonzero. Thus, we have

$$
\sum_{n \in Z_{j}(N)} \xi_{j}(n) \int_{m_{j}} f_{2}^{2}(\alpha) f_{3}^{2}(\alpha) f_{4}^{2}(\alpha) e(-\alpha n) d \alpha=\int_{m_{j}} f_{2}^{2}(\alpha) f_{3}^{2}(\alpha) f_{4}^{2}(\alpha) K_{j}(\alpha) d \alpha
$$

where the exponential sum $K_{j}(\alpha)$ is defined by

$$
K_{j}(\alpha)=\sum_{n \in Z_{j}(N)} \xi_{j}(n) e(-\alpha n)
$$

Let

$$
I_{j}=\int_{m_{j}} f_{2}^{2}(\alpha) f_{3}^{2}(\alpha) f_{4}^{2}(\alpha) K_{j}(\alpha) d \alpha
$$

By (3.1)-(3.3), we get

$$
I_{j} \geq \sum_{n \in Z_{j}(N)} n^{7 / 6} L^{-A} \gg Z_{j} N^{7 / 6} L^{-A} .
$$

Next, we will use the following lemma to compute $Z_{j}$.

Lemma 3.1. (see [11, Lemma 2.1] with $k=2$ or [5, (3.6)]) Let $f_{2}(\alpha)$ be defined in (2.3) and $K_{j}$ be defined above. Then

$$
\int_{0}^{1}\left|f_{2}(\alpha) K_{j}(\alpha)\right|^{2} d \alpha \ll N^{\varepsilon}\left(Z_{j} N^{1 / 2}+Z_{j}^{2}\right)
$$




\subsection{The integrals over $Z_{j}$}

We now establish our estimate for $Z_{j}$. An application of Cauchy-Schwarz inequality yields the inequality

$$
I_{1} \ll\left(\max _{\alpha \in \mathfrak{m}_{1}}\left|f_{3}^{2}(\alpha)\right|\right)\left(\int_{0}^{1}\left|f_{2}(\alpha) K_{1}(\alpha)\right|^{2} d \alpha\right)^{1 / 2}\left(\int_{0}^{1}\left|f_{2}(\alpha) f_{4}^{2}(\alpha)\right|^{2} d \alpha\right)^{1 / 2} .
$$

Combining Lemmas 2.2, 2.4 and 3.1 , we find that

$$
\begin{aligned}
I_{1} & \ll\left(N^{133 / 480+\varepsilon}\right)^{2}\left(N^{\varepsilon}\left(Z_{1} N^{1 / 2}+Z_{1}^{2}\right)\right)^{1 / 2}\left(N^{1+\varepsilon}\right)^{1 / 2} \\
& \ll N^{253 / 240+\varepsilon}\left(Z_{1}^{1 / 2} N^{1 / 4+\varepsilon}+Z_{1} N^{\varepsilon}\right) \ll Z_{1}^{1 / 2} N^{313 / 240+\varepsilon}+Z_{1} N^{253 / 240+\varepsilon} .
\end{aligned}
$$

Hence, (3.4) and (3.5) reveal that

$$
Z_{1} \ll N^{11 / 40+\varepsilon}
$$

We use the same method to compute $Z_{2}$, so

$$
I_{2} \ll\left(\max _{\alpha \in \mathfrak{m}_{2}}\left|f_{3}^{2}(\alpha)\right|\right)\left(\int_{0}^{1}\left|f_{2}(\alpha) K_{2}(\alpha)\right|^{2} d \alpha\right)^{1 / 2}\left(\int_{0}^{1}\left|f_{2}(\alpha) f_{4}^{2}(\alpha)\right|^{2} d \alpha\right)^{1 / 2} .
$$

Combining Lemmas 2.2, 2.5 and 3.1, we find that

$$
I_{2} \ll Z_{2}^{1 / 2} N^{115 / 84+\varepsilon}+Z_{2} N^{47 / 42+\varepsilon} .
$$

Hence, (3.4) and (3.7) reveal that

$$
Z_{2} \ll N^{17 / 42+\varepsilon}
$$

\section{Proof of Theorem 1.1}

Let $Z(N)$ denote the number of even integers $n$ in the interval $[N / 2, N]$ such that the following asymptotic formula

$$
R(n)=\frac{1}{576} \Theta(n) \Im(n)+O\left(n^{7 / 6} L^{-A}\right)
$$

fails to hold. On recalling $(3.6)$ and $(3.8)$, we arrive at the conclusion that

$$
Z(N) \ll Z_{1}+Z_{2} \ll N^{17 / 42+\varepsilon} .
$$

Hence $E(N) \ll N^{17 / 42+\varepsilon}$.

\section{Acknowledgments}

The author would like to thank Professor Guangshi Lü for his valuable advice and constant encouragement. Moreover, the author wishes to express his thanks to the referee for many useful suggestions and comments. 


\section{References}

[1] J. Brüdern, Sums of squares and higher powers I, J. London Math. Soc. (2) 35 (1987), no. $2,233-243$.

[2] _ On Waring's problem for two cubes and two small cubes, Acta Arith. 155 (2012), no. 3, 271-285.

[3] _ On the asymptotic formula in Waring's problem: one square and three fifth powers, Glasg. Math. J. 57 (2015), no. 3, 681-692.

[4] A. A. Karatsuba, Basic Analytic Number Theory, Springer-Verlag, Berlin, 1993.

[5] Y. Liu, Exceptional set for sums of unlike powers of primes, Int. J. Number Theory 15 (2019), no. 2, 339-352.

[6] Z. Liu, Goldbach-Linnik type problems with unequal powers of primes, J. Number Theory 176 (2017), 439-448.

[7] X. D. Lü, Waring-Goldbach problem: two squares, two cubes and two biquadrates, Chinese Ann. Math. Ser. A 36 (2015), no. 2, 161-174.

[8] C. D. Pan and C. B. Pan, Goldbach's Conjecture, Science Press, Beijing, 1981.

[9] R. C. Vaughan, On the representation of numbers as sums of powers of natural numbers, Proc. London Math. Soc. (3) 21 (1970), 160-180.

[10] _ The Hardy-Littlewood Method, Second edition, Cambridge Tracts in Mathematics 125, Cambridge University Press, Cambridge, 1997.

[11] T. D. Wooley, Slim exceptional sets and the asymptotic formula in Waring's problem, Math. Proc. Cambridge Philos. Soc. 134 (2003), no. 2, 193-206.

[12] _ On Waring's problem for intermediate powers, Acta Arith. 176 (2016), no. 3, 241-247.

[13] M. Zhang and J. Li, Exceptional set for sums of unlike powers of primes, Taiwanese J. Math. 22 (2018), no. 4, 779-811.

Xaodong Zhao

School of Mathematics, Shandong University, Jinan, Shandong 250100, P. R. China

E-mail address: xdzhao2016@163.com 